\title{
ON GROWTH IN GAUSSIAN ELIMINATION WITH COMPLETE PIVOTING*
}

\author{
NICK GOULD†
}

\begin{abstract}
It has been conjectured that when Gaussian elimination with complete pivoting is applied to a real $n$-by- $n$ matrix, the maximum possible growth is $n$. In this note, a 13 -by-13 matrix is given, for which the growth is 13.0205 . The matrix was constructed by solving a large nonlinear programming problem. Growth larger than $n$ has also been observed for matrices of orders 14,15 , and 16.
\end{abstract}

Key words. Gaussian elimination, growth, complete pivoting, nonlinear programming methods

AMS(MOS) subject classifications. 65F05, 65G05

1. Introduction. Let $A$ be an $n$-by- $n$ real matrix, let $A^{(1)}=A$, and let $A^{(k+1)}$, for $k=1, \cdots, n-1$, be the $n-k$-by- $n-k$ matrix derived from $A$ by elimination operations. That is, if we partition $A^{(k)}$ as

$$
A^{(k)}=\left(\begin{array}{cc}
\alpha^{(k)} & a_{c}^{(k) T} \\
a_{r}^{(k)} & A_{B}^{(k)}
\end{array}\right),
$$

(where the scalar $\alpha^{(k)}$ is known as the pivot at the $k$ th stage of the elimination), then

$$
A^{(k+1)}=A_{B}^{(k)}-a_{r}^{(k)}\left[\alpha^{(k)}\right]^{-1} a_{c}^{(k) T} .
$$

Alternatively, $A^{(k+1)}$ is the Schur complement of the first $k$-by- $k$ block of $A$ in the matrix $A$.

If Gaussian elimination, with complete or partial pivoting, is used to solve the system of linear equations $A x=b$, Wilkinson [9] showed that the computed solution $\hat{x}$ satisfies the perturbed equations

$$
(A+E) \hat{x}=b,
$$

where the error matrix $E$ satisfies the normwise bound

$$
\|E\|_{\infty} \leqq u p(n) g_{n}(A)\|A\|_{\infty} \text {. }
$$

Here $u$ is the unit roundoff, $p(n)$ is a cubic polynomial of $n$, and

$$
g_{n}(A)=\max _{\substack{1 \leqq i, j \leqq n-k+1, 1 \leqq k \leqq n}}\left|(P A Q)_{i j}^{(k)}\right| / \max _{1 \leqq i, j \leqq n}\left|(P A Q)_{i j}\right|,
$$

where $P$ and $Q$ represent the pivoting permutations applied to $A$ during the elimination. As the other contributions to the bound (1.4) are beyond our control, it is of interest to know precisely how large the growth factor $g_{n}(A)$ can be.

We say that $A$ is a complete elimination matrix if, at each stage of the elimination, the modulus of each entry in $A^{(k)}$ is no larger than that of the pivot. Such matrices arise when complete pivoting is used to permute the rows and columns of a general matrix during Gaussian elimination (see Wilkinson [11]). The permutation matrices $P$ and $Q$ in (1.5) are both the identity matrix if $A$ is a complete elimination matrix. Moreover, 1990.

* Received by the editors September 7, 1990; accepted for publication (in revised form) November 7,

† Central Computing Department, Rutherford Appleton Laboratory, Chilton, Oxfordshire, OXI1 0QX, England (nimg@ib.rl.ac.uk). 
the growth factor is now the ratio of the moduli of the largest pivot to the first. For such matrices, Wilkinson [9] showed that

$$
g_{n}=\sup _{A} g_{n}(A)<n^{1 / 2}\left(23^{1 / 2} 4^{1 / 3} \cdots n^{1 / n-1}\right)^{1 / 2}
$$

and he noted that there were no known examples of matrices for which $g_{n}(A)>n$ (Wilkinson, [10, p. 97] and [11, p. 213]). Indeed, Cryer [3] hypothesized that $g_{n} \leqq n$ for all $n$ with equality if and only if there is a Hadamard matrix, that is a matrix with entries \pm 1 and orthogonal rows and columns, of dimension $n$. Higham and Higham [6] give a class of matrices for which $g_{n}(A) \geqq(n+1) / 2$, while simulations by Trefethen and Schreiber [8] on random matrices indicate average growths on random matrices of approximately $n^{1 / 2}$.

One way of trying to generate large growth factors for complete elimination matrices is to attempt to solve the optimization problem of maximizing the modulus of the $n$th pivot. (It is always possible to arrange that the maximum growth occurs at this pivot. For, suppose the $k$ th pivot is largest in magnitude. Then the matrix formed by replacing the last $k$-by- $k$ block of the $n$-by- $n$ identity matrix, scaled by $a_{11}$, with the first $k$-by- $k$ block of $A$ is also a complete elimination matrix with the same growth factor but with the maximum growth now occurring at the $n$th pivot.) This approach has been considered by Day and Peterson [4] and is also the approach taken in this note. Day and Peterson give lower bounds on the growth for $1 \leqq n \leqq 8$. Here we extend the range to $1 \leqq n \leqq$ 16. The major result we obtain is that there are a number of 13-by-13 matrices for which $g_{13}$ is larger than 13, and thus that Cryer's conjecture is false. Examples of growth larger than $n$ have also been observed for matrices of order 14, 15, and 16.

In $\S 2$, we describe the nonlinear programming approach we have taken to this problem. In $\S 3$, the results of our numerical experiments are presented. We give an example where $g_{13}>13$ in the Appendix.

2. Method. We may formulate the maximum pivot growth problem as a nonlinear optimization problem as follows:

Starting with an $n$-by- $n$ real matrix $X^{(1)}=A$, we let $X^{(k)}$ be the matrix

$$
X^{(k)}=\left(\begin{array}{cc}
0 & 0 \\
0 & A^{(k)}
\end{array}\right)
$$

where $A^{(k)}$ is the $k$ th elimination matrix (1.1). Let $x_{i, j, k}$ be the $(i, j)$ th entry of $X^{(k)}$. We thus wish to maximize $x_{n, n, n}$ subject to the restrictions that the matrices $X^{(k)}$ and $X^{(k+1)}$ are related to each other by elimination operations, that the largest element in $X^{(k)}$ occurs in position $(k, k)$, and that the initial matrix $X^{(1)}$ is scaled so that the largest entry in magnitude is 1 . This leads to the problem

$$
\text { maximize } x_{n, n, n}
$$

subject to the elimination constraints:

$$
\begin{aligned}
& x_{i, j, k+1}-x_{i, j, k}+x_{i, k, k} x_{k, j, k} / x_{k, k, k}=0, \\
& \text { for } k<i, j \leqq n \text { and } k=1, \cdots, n-1 ;
\end{aligned}
$$

constraints which make the signs of the pivots unique:

$$
x_{k, k, k} \geqq 0 \quad \text { for } \quad k=1, \cdots, n ;
$$

a normalizing constraint, $x_{1,1,1}=1$; and complete pivoting constraints:

$$
-1 \leqq x_{i, j, 1} \leqq 1 \text { for } 1 \leqq i, j \leqq n
$$


and

$$
-x_{k, k, k} \leqq x_{i, j, k} \leqq x_{k, k, k} \quad \text { for } \quad k \leqq i, j \leqq n \quad \text { and } \quad k=2, \cdots, n-1 .
$$

This formulation involves roughly $n^{3} / 3$ variables, but is a very sparse optimization problem. We chose to solve this problem using our large-scale nonlinear programming package, LANCELOT (Conn, Gould, and Toint [2]), since the package is designed to handle such nonlinear sparsity as appears in problem (2.2)-(2.6) above.

By contrast, Day and Peterson [4] formulate the problem entirely in terms of the $n^{2}$ variables $X^{(1)}$, treating all of the remaining variables $X^{(k)}, k=2, \cdots, n$ as implicit functions of $X^{(1)}$. This leads to a problem that is significantly more nonlinear and makes the calculation of analytic derivatives considerably harder. Nonetheless, Day and Peterson report considerable success with the nonlinear programming package NPSOL (Gill, Murray, Saunders, and Wright [5]).

Of course, neither nonlinear programming method is designed to find anything stronger than local solutions to a problem. The problem $(2.2)-(2.6)$ has many local solutions and most of them are highly degenerate. The problem is thus challenging for a nonlinear programming algorithm and the values given in the next section are the result of many runs from different starting points in an attempt to find the global solution to the problem.

3. Results. In Table 3.1 , we give the results obtained by running LANCELOT on the problem posed in $\S 2$. LANCELOT is written in standard Fortran 77, compiled in double precision with the SUN Fortran 1.3 compiler; the problems were solved on a SUN SPARCstation 1. Each problem has many local solutions; we cannot, of course, guarantee that the values reported are the largest growths that can be obtained, merely that they are the largest values we encountered.

Of particular interest are the values obtained for $n=13,14,15$, and 16, for here we see growth of more than $n$. We also observe that for $n=16$, where a complete elimination Hadamard matrix exists and gives rise to growth of 16 , other complete elimination matrices give larger growth. Thus Cryer's [3] conjecture is false.

TABLE 3.1

Maximum growth factors encountered.

\begin{tabular}{rll}
\hline$n$ & Growth size & \multicolumn{1}{c}{ Comments } \\
\hline 1 & 1.0 & trivial \\
2 & 2.0 & trivial \\
3 & 2.25 & optimal (see Cohen, [1]) \\
4 & 4.0 & Hadamard matrix, optimal (see Cryer [3]) \\
5 & 4.1325 & agrees with Day and Peterson [4] \\
6 & 5.0 & agrees with Day and Peterson [4] \\
7 & 6.0 & agrees with Day and Peterson [4] \\
8 & 8.0 & Hadamard matrix \\
9 & 8.4305 & \\
10 & 9.5294 & \\
11 & 10.4627 & \\
12 & 12.0 & \\
13 & 13.0205 & Hadamard matrix \\
14 & 14.5949 & \\
15 & 16.1078 & \\
16 & 18.0596 & not a Hadamard matrix \\
\hline
\end{tabular}


The matrices that give rise to the growth factors reported in Table 3.1 are often extremely sensitive to small perturbations in their entries in that tiny perturbations to a complete elimination matrix rarely results in another such matrix. This phenomenon was observed by Day and Peterson [4] and may explain why examples of large growth have proved elusive in previous attempts to find them. It also makes it rather difficult to specify matrices which give rise to large growth. Indeed, we had to solve the optimization problem of $\S 2$ to very high accuracy, requiring the residuals of the nonlinear constraints (2.3) to be of the order of the unit roundoff. In some cases, this meant that we had to take the best solution that we obtained on the SUN as a starting point for a further run in extended precision on the CRAY X-MP/416 at Rutherford to reduce the residuals to the desired level. Even then, the mere fact of rounding the CRAY values to 16 decimal places frequently prevented the computed matrix from being a complete elimination matrix when the operations (1.2) were performed in double precision on the SUN. The values obtained had to be adjusted by eye to obtain a suitable floating-point complete elimination matrix.

We specify a 13-by-13 matrix that gives rise to growth of slightly more than 13.0205 , in IEEE double precision arithmetic on a SUN SPARCstation 1, in the Appendix to this paper. The values must be read in Fortran 1P,D24.16 format. It is not known whether there are matrices with simple fractional entries that give rise to such large growth. Other 13-by-13 matrices that give rise to growth of larger than 13 were encountered.

The results of applying the elimination operations (1.2) to this matrix are given in Table 3.2. The size of the pivot and the largest nonpivot in absolute value at each stage of the elimination are shown. Note that the pivots are far from monotonic and that there is a "surge" of growth in the last few stages. Such a surge has been observed for Hadamard matrices by other authors [3], [4]. Indeed, values for the last six pivots for such matrices are known [4, Prop. 5.5]. Also observe how close the largest nonpivot at each stage is to the pivot and thus how tiny perturbations to the matrix elements may completely alter the pivot sequence.

4. Conclusions. We have shown that growth of larger than $n$ is possible when Gaussian elimination with complete pivoting is performed on real $n$-by- $n$ matrices by exhibiting a 13-by-13 matrix for which this is true. If $A$ is an $n$-by- $n$ complete elimination

TABLE 3.2

Details of the elimination.

\begin{tabular}{rrr}
\hline Pivot & Pivot size & $\begin{array}{c}\text { Largest modulus of } \\
\text { nonpivot entry }\end{array}$ \\
\hline 1 & 1.0000000000000000 & 1.0000000000000000 \\
2 & 2.0000000000000000 & 2.0000000000000000 \\
3 & 2.0000000000000000 & 2.0000000000000000 \\
4 & 2.5964300000000002 & 2.5964300000000002 \\
5 & 2.3776999999999999 & 2.3776999543751263 \\
6 & 2.3038700000000003 & 2.3038700000000003 \\
7 & 2.9587400000000001 & 2.9587398634283884 \\
8 & 3.5890399999999998 & 3.5890399999999998 \\
9 & 4.1163800000000004 & 4.1163800000000004 \\
10 & 3.3550400000000007 & 3.3550399999999998 \\
11 & 6.5102699999999984 & 6.5102698773166514 \\
12 & 6.5102700000000011 & 6.5102699999999567 \\
13 & 13.0205000013724188 & - \\
\hline
\end{tabular}


matrix with growth $g(A)$, and $P$ is the matrix which permutes the first $2 n$ integers to $\{1, n+1,2, n+2, \cdots, n, 2 n\}$, then (see [7] and [4, Prop. 5.12])

$$
P^{T}\left(\begin{array}{rr}
A & A \\
A & -A
\end{array}\right) P
$$

is also a complete elimination matrix with growth $2 g(A)$. Thus there are an infinite number of matrices, of dimensions $13 \cdot 2^{k}$ for nonnegative $k$, which give rise to growth larger than their dimension. We suspect that there are examples of large growth for many other dimensions-we have encountered such examples for $n=14,15$, and 16and that

$$
\limsup _{n \rightarrow \infty} g_{n} / n
$$

is unbounded. It is not known if there are matrices of dimension smaller than 13 for which growth larger than $n$ is possible, nor is it known quite how close the growth factors given in this paper are to $g_{n}$.

We have observed that examples of large growth in complete elimination are very unstable in that very small perturbations to the matrix entries give rise to radically different pivot sequences. We suspect that this is why such examples have not been observed in practice. We also realize that the examples given here are extremely unlikely to-nor indeed should they-discourage people from using Gaussian elimination with pivoting. The potentially less stable partial and threshold pivoting strategies are used with impunity, and considerable success, throughout the scientific world.

Appendix. Here we give a 13-by-13 complete elimination matrix for which the growth is slightly over 13.0205 when the elimination operations (1.2) are performed in IEEE double precision arithmetic on a SUN SPARCstation 1. The values should be read in Fortran 1P,D24.16 format.

row 1

$$
\begin{array}{rrr}
1.0000000000000000 D+00 & -1.0000000000000000 D+00 & -1.0000000000000000 D+00 \\
6.6084891857885364 D-01 & 3.5076867724029653 D-01 & 1.3913093634808771 D-01 \\
1.0000000000000000 D+00 & -1.0000000000000000 D+00 & 9.4546309508853699 D-01 \\
-6.4358761317393848 D-02 & -4.7259056539260776 D-02 & 9.8144752878695718 D-01 \\
1.0000000000000000 D+00 & &
\end{array}
$$$$
\text { row } 2
$$$$
\begin{array}{rrr}
1.0000000000000000 D+00 & 1.0000000000000000 D+00 & -1.0000000000000000 D+00 \\
-1.0000000000000000 D+00 & -8.8262544148845457 D-01 & -7.9349789219584022 D-01 \\
-1.0000000000000000 D+00 & -7.0049633754068708 D-01 & 1.0000000000000000 D+00 \\
1.0000000000000000 D+00 & -1.0000000000000000 D+00 & 1.0000000000000000 D+00 \\
-6.5149858941930272 D-01 & &
\end{array}
$$ 
row 4

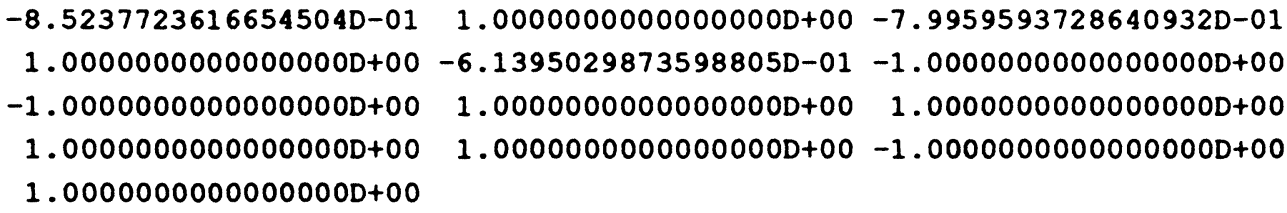


row 10

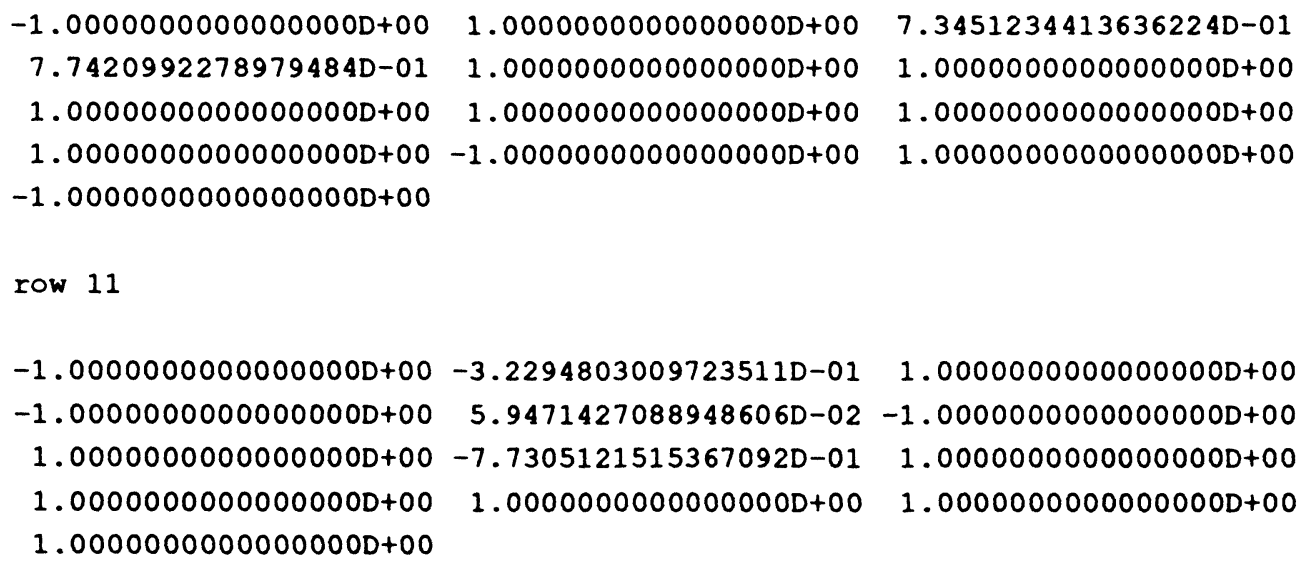

Acknowledgment. The author would like to thank two other Nicks (Higham and Trefethen) for stimulating discussions on this topic over the years and Iain Duff for his helpful comments on this paper. He is also grateful to Philippe Toint and Andy Conn for their interest in the problem and collaboration on the LANCELOT project. Finally, thanks are due to the associate editor and referees for the prompt and efficient processing of the paper.

\section{REFERENCES}

[1] A. M. CoHEN, A note on the pivot size in Gaussian elimination, Linear Algebra Appl., 8 (1974), pp. 361-368.

[2] A. R. CONN, N. I. M. GoulD, AND PH.L. TOINT, An introduction to the structure of large scale nonlinear optimization problems and the LANCELOT project, FUNDP Report 89/19, Facultés Universitaires Notre-Dame de la Paix, Namur, Belgium, 1989; Also in Proc. 9th Internat. Conference on Computing Methods in Applied Sciences and Engineering, Paris, 1990.

[3] C. W. CRYER, Pivot growth in Gaussian elimination, Numer. Math., 12 (1968), pp. 335-345. 
[4] J. DAy AND B. Peterson, Growth in Gaussian elimination, Amer. Math. Monthly, June, 1988, pp. 489513.

[5] P. E. Gill, W. Murray, M. A. SAunders, and M. H. Wright, User's guide for SOL/NPSOL: $A$ Fortran package for nonlinear programming, Tech. Report SOL 83-12, Systems Optimization Laboratory, Stanford University, Stanford, CA, 1983.

[6] N. J. HIGHAM AND D. J. HIGHAM, Large growth factors in Gaussian elimination with pivoting, SIAM J. Matrix Anal. Appl., 10 (1989), pp. 155-164.

[7] L. TORNHEIM, Maximum pivot size in Gaussian elimination with complete pivoting, Tech. Report, Chevron Research Company, Richmond, CA, 1970.

[8] L. N. TREFETHEN AND R. S. SCHREIBER, Average-case stability of Gaussian elimination, SIAM J. Matrix Anal. Appl., 11 (1990), pp. 335-360.

[9] J. H. Wilkinson, Error analysis of direct methods of matrix inversion, J. Assoc. Comput. Mach., 8 (1961), pp. 281-330.

[10] - Rounding errors in algebraic processes, Notes on Applied Science No. 32, 1963, Her Majesty's Stationery Office, London.

[11] — , The Algebraic Eigenvalue Problem, Oxford University Press, London, 1965. 